\title{
вмJ Global Health From the battlefield to the bedroom: a multilevel analysis of the links between political conflict and intimate partner violence in Liberia
}

\author{
Jocelyn T D Kelly, ${ }^{1}$ Elizabeth Colantuoni, ${ }^{2}$ Courtland Robinson, ${ }^{3}$ Michele R Decker ${ }^{4}$
}

\begin{abstract}
To cite: Kelly JTD, Colantuoni E, Robinson C, et al. From the battlefield to the bedroom: a multilevel analysis of the links between political conflict and intimate partner violence in Liberia. BMJ Glob Health 2018;3:e000668. doi:10.1136/ bmjgh-2017-000668
\end{abstract}

Handling editor Seye Abimbola

Received 9 December 2017 Revised 5 March 2018 Accepted 6 March 2018

Check for updates

${ }^{1}$ Department of Population, Family and Reproductive Health, Johns Hopkins University Bloomberg School of Public Health, Baltimore, Maryland, USA

${ }^{2}$ Department of Biostatistics, Johns Hopkins Bloomberg School of Public Health, Baltimore, Maryland, USA ${ }^{3}$ Department of International Health, Johns Hopkins Bloomberg School of Public Health, Baltimore, Maryland, USA

${ }^{4}$ Department of Population, Family and Reproductive Health, Women's Health and Rights Program, Center for Public Health and Human Rights, Johns Hopkins Bloomberg School of Public Health, Baltimore, Maryland, USA

Correspondence to Dr Jocelyn T D Kelly; jtdkelly@gmail.com

\section{ABSTRACT}

Objectives Assess the link between levels of armed conflict and postconflict intimate partner violence (IPV) experienced by women in Liberia.

Methods Armed Conflict Location and Event Data Project data were used to measure conflict-related fatalities in districts in Liberia during the country's civil war from 1999 to 2003. These data were linked to individual-level data from the 2007 Demographic and Health Survey, including past-year IPV. Multilevel logistic models accounting for the clustering of women within districts evaluated the relationship of conflict fatalities with postconflict past-year IPV. Additional conflict measures, including conflict events and cumulative years of conflict, were assessed.

Results After adjusting for individual-level characteristics correlated with IPV, residence in a conflict fatality-affected district was associated with a $50 \%$ increase in risk of IPV (adjusted OR (aOR): 1.55, 95\% Cl 1.26 to 1.92). Women living in a district that experienced $4-5$ cumulative years of conflict were also more likely to experience IPV (aOR 1.88, $95 \% \mathrm{Cl} 1.29$ to 2.75 ).

Conclusion Residing in a conflict-affected district even 5 years after conflict was associated with postconflict IPV. Policy implications Recognising and preventing postconflict IPV violence is important to support long-term recovery in postconflict settings.

\section{INTRODUCTION}

Each year, war and interpersonal violence account for a significant burden on morbidity and mortality worldwide. Currently, levels of political violence are among the highest since World War II, with worldwide conflicts increasing dramatically since 2012. ${ }^{1}$ Recent studies have shown how violence can spread across populations temporally and spatially, yet the association between armed conflict and postconflict interpersonal violence is poorly documented. One form of interpersonal violence that is increasingly linked with conflict-related violence is violence against women in the conflict or postconflict phase. $^{2-5}$ A new frontier in understanding conflict involves quantifying how political

\section{Key questions}

What is already known?

- Currently, levels of political violence are among the highest since World War II, with worldwide conflicts increasing dramatically since 2012.

- Each year, war and interpersonal violence account for a significant burden on morbidity and mortality worldwide.

- One form of interpersonal violence that is increasingly linked with conflict-related violence is intimate partner violence (IPV) in the postconflict phase.

\section{What are the new findings?}

- This research would be the first to our knowledge published in the peer-reviewed literature to use multilevel modelling to examine the relationship between residing in a place affected by political conflict, and violence against women years after peace is declared.

- This research finds that residence in a conflict fatality-affected district was associated with a 50\% increase in risk of IPV, after adjusting for individuallevel characteristics normally correlated with this type of violence.

- Women living in a district that experienced 4-5 cumulative years of conflict were almost $90 \%$ more likely to experience IPV than a counterpart living in a district with no conflict.

What do the new findings imply?

- Governments, clinicians and service providers should explicitly screen for and provide programmes to address domestic violence after conflict.

violence may impact human aggression even after formal peace is declared.

Thirty-five per cent of women globally report being victims of physical or sexual abuse during their lifetime and one-third of women who have been in a relationship have experienced physical or sexual violence from a partner. ${ }^{6}$ Globally, intimate partner violence 
(IPV) is the most common form of violence against women. ${ }^{7}$ Sequelae of this violence include injury and death as well as poorer mental and reproductive health outcomes,${ }^{7-9}$ including unintended pregnancy, physical harm, increased vulnerability to sexually transmitted diseases and HIV. ${ }^{10-14}$

Research from Uganda, Thailand, Cote D'Ivoire and Liberia has found that women who have higher levels of conflict-related abuses also report higher levels of IPV victimisation during and after conflict. ${ }^{2-5}$ Exposure to political violence and to human rights abuses at the individual level has been linked to higher rates of IPV perpetration among men in conflict and postconflict settings. ${ }^{451516}$ Thus, personal experience with conflict-related abuse appears to enhance vulnerability to IPV.

However, the indirect impact of conflict on violence remains less clear. Far fewer studies have examined how living in a conflict-affected place affects IPV. An unpublished study from Peru found that conflict violence at the provincial level was associated with IPV risk among women. ${ }^{17}$ Similar to the current study, Gallegos and Gutierrez combined Demographic and Health Survey (DHS) data with conflict data from the Peruvian Truth and Reconciliation Commission. Multilevel modelling revealed that women exposed to a conflict event had a greater probability of being a victim of IPV 5 years or more after conflict. Janko et al presented a conference abstract a study set in Rwanda, which found that proximity to conflict deaths significantly increased IPV risk. ${ }^{18}$ Across this emergent body of literature, conflict-related measures range from both fatal and non-fatal conflict events ${ }^{17}$ to a focus solely on fatalities. ${ }^{18}$ While the pathways for this 'contagion' effect remain unclear, these studies, from very different contexts, suggest that conflict may have both direct and indirect pathways for influencing the levels of violence after conflict.

The current study extends this literature by taking a multilevel modelling approach to understand how conflict-related violence is associated with IPV after conflict, and directly compares conflict measures to examine how aspects of conflict relate to IPV.

\section{Setting}

Liberia offers the ability to examine the impact of armed conflict at the district level and postconflict individual-level IPV due to availability of data describing both conflict-related violence and postconflict health outcomes. Liberia suffered two successive and devastating civil wars from 1989 to 1997 and from 1999 to $2003 .{ }^{19} 20$ In 2003, after international intervention, a peace agreement was signed and rebel troops were demobilised. Over 150000 people died during this second conflict and resulted in the displacement of 850000 refugees into bordering nations. ${ }^{21}$

\section{METHODS}

\section{Analytic sample}

This study combines individual-level data from the Liberia 2007 DHS, with Armed Conflict Location and Event Data Project (ACLED) data using unique geographic identifying codes from both data sets. For the 2007 Liberia DHS, a total of 7448 women were sampled, with 7092 women completing the survey, a $95 \%$ response rate. A total of 4913 women aged 15-49 were administered the Domestic Violence (DV) Module. Eight per cent of these women $(n=411)$ were dropped due to missing the necessary geographic identifier, leaving 4502 women. Of this sample, only ever-partnered women were asked questions about IPV ( $\mathrm{n}=3648)$. Women responding that they had ever experienced abuse were then asked if they had experienced abuse in the past 12 months. Over $98 \%$ of the sample ( $\mathrm{n}=3596$ women) responded to this question and are thus eligible for inclusion in the analysis. This analysis used a complete case method. Roughly $3 \%$ of the sample had missing values for relevant exposures in our analysis and were dropped from the analysis. Because of the very low level of missing data, however, no replacement or imputation was used. Of the 3596 original cases with data on IPV, $3452(96.6 \%)$ were used in this analysis.

\section{Outcome: IPV}

The DHS DV Module measures IPV using a modified Conflict Tactics Scale, a widely used measure with strong psychometric properties. ${ }^{22}$ Specifically, ever-partnered women were asked about a list of eight specific behaviours, including shaking, slapping and punching, they may have experienced that would classify as physical or sexual violence. For those items where women answered 'yes', they were then asked about the frequency of the act in the 12 months preceding the survey. Past-year IPV is defined as having experienced any of the eight behaviours in the past 12 months.

\section{Exposures: measures of conflict}

There are 61 districts in Liberia; over the course of the conflict, nine of these districts experienced fatalities $(14.7 \%)$. There were 182.4 fatalities per district on average, but a notably large range of values (range 1-327, SD 113.4). Thirty-nine of the 61 districts experienced conflict-related events $(63.9 \%)$. Of these districts, there were 41.9 average events per district (range 1-134, SD 54.0). Seventy-six per cent of individuals in the data set $(\mathrm{n}=3734)$ lived in districts with conflict events, compared with 33.3\% ( $\mathrm{n}=1497)$ who lived in districts with fatalities.

ACLED provides two measures of political conflict: events and fatalities from 1999 to 2003. Events are coded as any recorded political incident, including clashes, protests, riots and battles. Fatalities are coded when an event results in one or more deaths. Every conflict fatality represents a political event, but not all events result in fatalities. The existence of two measures raises the question of which measure to use as the primary exposure for conflict and how best to characterise it. 
Fatalities are concrete, visible and disturbing manifestations of conflict, and therefore serve as the primary exposure of this analysis. The relatively limited number of fatalities per district means that this measure was kept as a dichotomous measure (any vs no fatalities). A sensitivity analysis was also conducted using events, which are not necessarily violent and may be less traumatic, as the exposure. Events were treated as both a dichotomous measure and an ordinal measure (no, medium and high). Finally, the cumulative years of impact was computed as another measure of exposure to conflict. Each district was classified as having no years, 1 year or multiple years of the conflict. This measure is used to assess whether the impact of conflict might result from an accumulating burden of conflict experiences rather than a simple count of experiences.

\section{Individual-level potential confounding variables}

Potential confounding variables measured for each woman were selected based on theory as well as by those variables that have been found to be significantly associated with interpersonal violence. Demographic variables included: age, number of children under 5, educational attainment and religion. Wealth and employment characteristics were: DHS wealth quintile measures and women's self-report of having worked in the past 12 months. Marriage and partner characteristics were: women's civil status (married, cohabiting or widowed/ divorced), partner's educational attainment and whether the partner uses alcohol (yes/no).

Attitudes towards violence and previous experience with violence were assessed through a single item that combined responses from three questions in the DHS. Respondents were asked whether their father had beaten them, and whether their father had beaten their mother. Finally, permissive attitudes towards IPV were assessed through a 5-item scale. In accordance with other similar studies, the 5-item assessment was collapsed into a variable that takes a value of 1 if women think beating is justified for any reason, and 0 if she thinks wife beating is never justified. ${ }^{17}$

\section{Model specification}

Multilevel logistic regression models were used to quantify the association between IPV and district-level conflict, that is, conflict-related fatalities (any vs none), after sequentially adding blocks of potential confounding variables to adjust for the characteristics of the women that comprise the district. The first block of variables consisted of demographic information and marriage characteristics including: age, number of children under 5, educational attainment, religion, marital status, partner's educational attainment and age married. The second block consisted of economic variables: wealth quintile and employment status. Finally, the last block accounted for two known correlates of violence: an aggregate measure of previous experience with violence and partner's alcohol use. The regression models included a random intercept

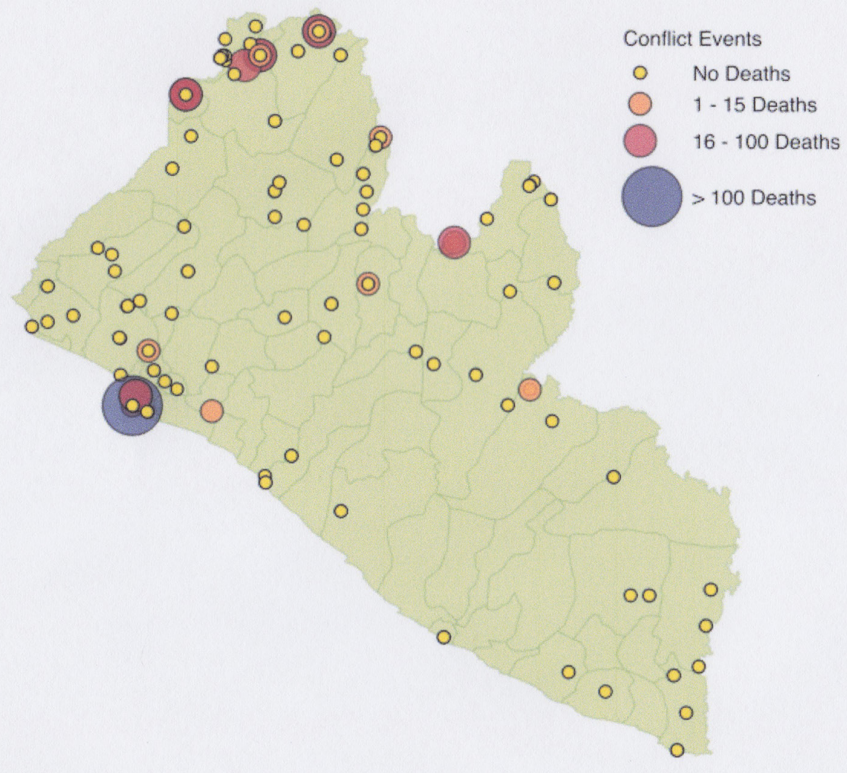

Figure 1 Conflict events and fatalities in Liberia during the Second Civil War.

for district to account for the clustering of IPV among women from the same district. Sensitivity analyses were conducted to look at the effect of changing the primary exposure from fatalities to conflict events (any vs none and no, medium and high levels) and cumulative conflict years.

To account for the possibility of migration diluting potential district-level effects, a sensitivity analysis was conducted, limiting the sample to those who had not moved since the start of the political violence. Thus, the effect of living in a conflict-affected district is hypothesised to be strongest among those who remained in the same district during and after the civil war.

All analyses were conducted with Stata/SE V.14.0. ${ }^{23}$ To take into account the complex survey design of the DHS, the survey weights for the DV Module were included in all analyses using the probability weight or pweight option within the gllamm command. ${ }^{24}$

\section{RESULTS}

Figure 1 illustrates how fatalities and events spread across space and time. Table 1 presents the district-level characteristics of the women included in the sample, disaggregated by residence in a fatality-affected district.

Table 2 presents the results of the stepwise model fitting. The unadjusted odds of women reporting IPV when living in a district with district-level conflict fatalities are two times the odds of reporting IPV when living in a district with no conflict-related fatalities (95\% CI 1.60 to 2.76). The association was not sensitive to accounting for basic demographics of the women living in the districts, including age, number of children under 5 , educational attainment and religion; intimate partner characteristics (adjusted OR (aOR) 2.08, 95\% CI 1.69 to 2.56) and economic characteristics (aOR 2.07, 95\% CI 1.70 to 2.53) 
Table 1 District-level summary measures of key demographic variables

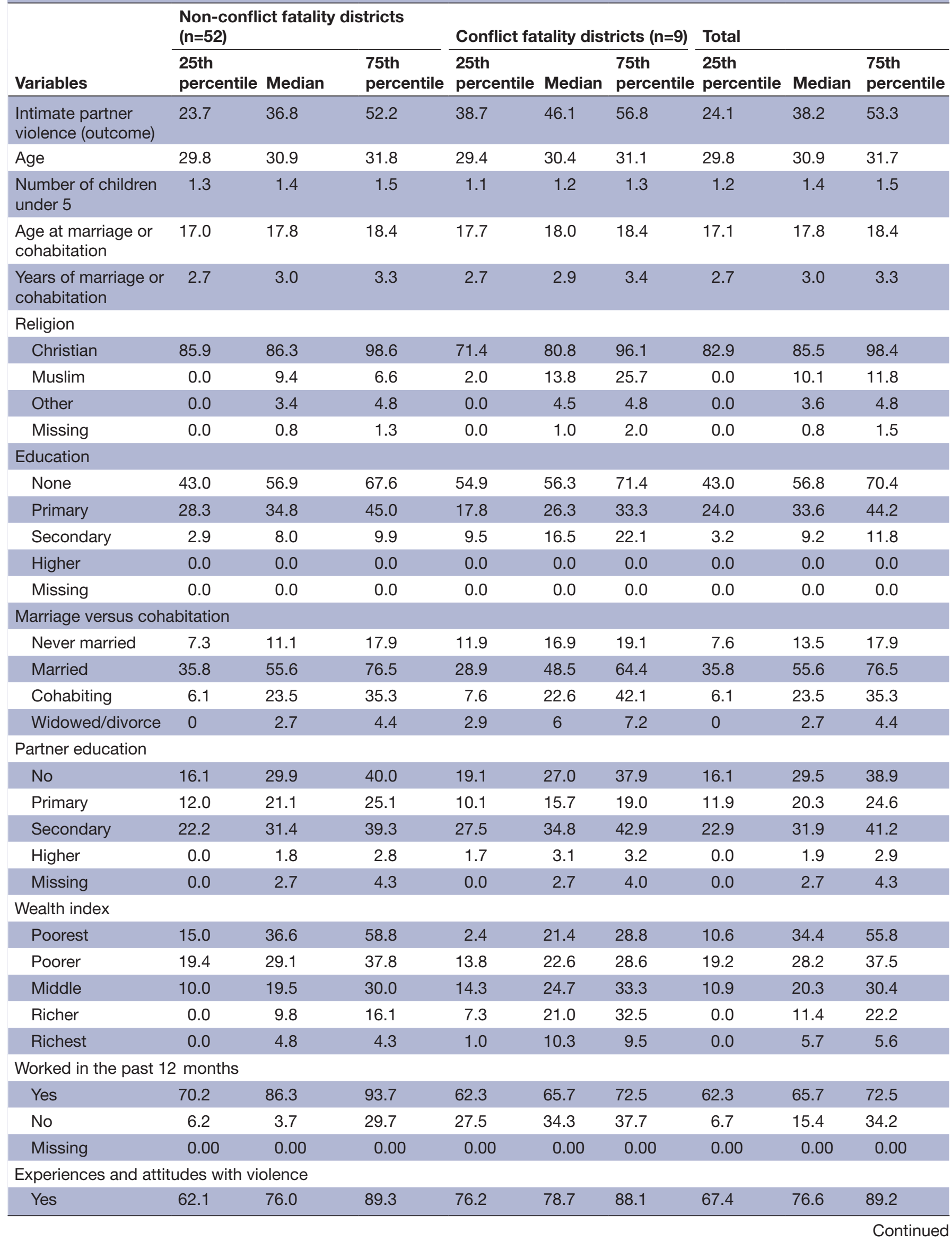




\begin{tabular}{|c|c|c|c|c|c|c|c|c|c|}
\hline \multirow[b]{2}{*}{ Variables } & \multicolumn{3}{|c|}{$\begin{array}{l}\text { Non-conflict fatality districts } \\
(n=52)\end{array}$} & \multicolumn{3}{|c|}{ Conflict fatality districts $(n=9)$} & \multicolumn{3}{|l|}{ Total } \\
\hline & $\begin{array}{l}\text { 25th } \\
\text { percentile }\end{array}$ & Median & $\begin{array}{l}\text { 75th } \\
\text { percentile }\end{array}$ & $\begin{array}{l}\text { 25th } \\
\text { percentile }\end{array}$ & Median & $\begin{array}{l}\text { 75th } \\
\text { percentile }\end{array}$ & $\begin{array}{l}\text { 25th } \\
\text { percentile }\end{array}$ & Median & $\begin{array}{l}\text { 75th } \\
\text { percentile }\end{array}$ \\
\hline No & 10.7 & 24.0 & 37.9 & 11.9 & 21.3 & 23.8 & 10.8 & 23.4 & 32.6 \\
\hline Missing & 0.0 & 0.0 & 0.0 & 0.0 & 0.0 & 0.0 & 0.0 & 0.0 & 0.0 \\
\hline \multicolumn{10}{|c|}{ Partner uses alcohol } \\
\hline Yes & 24.2 & 30.6 & 40.3 & 26.1 & 31.4 & 35.3 & 24.6 & 31.3 & 38.5 \\
\hline No & 45.9 & 54.0 & 64.3 & 41.2 & 49.4 & 54.9 & 45.3 & 53.8 & 61.3 \\
\hline Missing & 6.9 & 11.1 & 19.0 & 11.9 & 16.9 & 21.4 & 7.4 & 11.8 & 20.0 \\
\hline
\end{tabular}

also resulted in only small changes in the adjusted association. Finally, after additionally accounting for previous experiences with violence and partner's alcohol use, the adjusted association decreased (aOR 1.55, 95\% CI 1.26 to 1.92); however, the size of the association and statistical significance remained.

\section{Sensitivity analysis for additional conflict measures}

Table 3 summarises the association between IPV and the alternative measures used to characterise conflict. After accounting for all of the potential confounding variables, there was no significant association between IPV and the presence of conflict-related events when considering any versus no conflict-related events (aOR 1.17, 95\% CI 0.85 to 1.59 ) or the number of events (mid vs no: aOR $1.28,95 \%$ CI 0.89 to 1.84 , high vs no: aOR $1.03,95 \%$ CI 0.67 to 1.59$)$. However, individuals in districts experiencing the highest number of event-years (3 or more event-years) were significantly more likely to experience IPV compared with those in districts with no event-years (aOR $1.88,95 \%$ CI 1.29 to 2.75 ). This relationship did not reach significance at the 2-3year level (aOR 1.20, $95 \%$ CI 0.83 to 1.75 ) or 1-year level (aOR 1.31, 95\% CI 0.91 to 1.89$)$.

\section{Sensitivity analysis restricted to non-migrants}

Over two-thirds of the sample $(79.2 \%, \mathrm{n}=2752)$ lived in a conflict district, and remained there from the time of the conflict until the survey was conducted. To measure whether the effect of postconflict migration affected the primary model, a sensitivity analysis was done with only non-migrants. We see a stronger association (aOR 1.79, $95 \%$ CI 1.13 to 2.82 ) for the non-migrant model than for the same model with the full population (aOR 1.55, $95 \%$ CI 1.26 to 1.92 ).

\section{DISCUSSION}

The impact of political conflict on future interpersonal violence has implications for a country's ability to achieve lasting peace and prosperity. This research suggests that living in a district that experienced conflict fatalities during war is associated with increased risk of experiencing interpersonal violence in the postconflict period. A woman living in a Liberian district exposed to any conflict-related fatalities was more than $50 \%$ more likely to experience recent IPV than a woman living in a no-fatality district (aOR $1.55,95 \%$ CI 1.26 to 1.92 ). These findings support the hypothesis that political violence during conflict is associated with higher levels of IPV after conflict, even after adjusting for relevant partner-level and individual-level characteristics. The largest change on the effect estimate occurred in the final stepwise fitting procedure when previous experiences with violence and partner's alcohol use are added to the model, suggesting that these factors may account for a portion of the excess IPV risk in fatality-affected districts.

In the analysis of event-years, women living in districts with 4-5years of conflict events were almost twice as likely to experience IPV compared with women living in no-event districts (aOR 1.88 , 95\% CI 1.29 to 2.75 ). This may be partially explained by the fact that the districts with the very highest number of fatalities were clustered in the highest event-year category. Districts with lower fatalities were clustered in the lower event-year categories. However, these results do suggest a possible doseresponse relationship, with the highest fatality districts carrying more IPV risk. Unfortunately, the small number of districts with fatalities makes it impossible to examine a measure of low, medium and high fatalities in this analysis. There was no significant association between IPV and conflict-related events (both any vs none and no, medium and high)

Sensitivity analysis with non-migrants revealed an even stronger effect estimate than the primary analysis. Non-migrant women living in a fatality-affected district were 1.8 times as likely to experience IPV compared with a non-migrant counterpart living in a non-fatality district (aOR 1.79 , 95\% CI 1.13 to 2.82 ), suggesting that the impact of conflict may be strongest when individuals have a longer exposure to conflict-affected environments.

It may seem striking that conflict is associated with such a large increase in human aggression even 5 years after conflict has ended. However, a cluster randomised survey conducted 7 years after the end of the war in Liberia found that intimate partner physical violence was significantly associated with exposure to war events in 


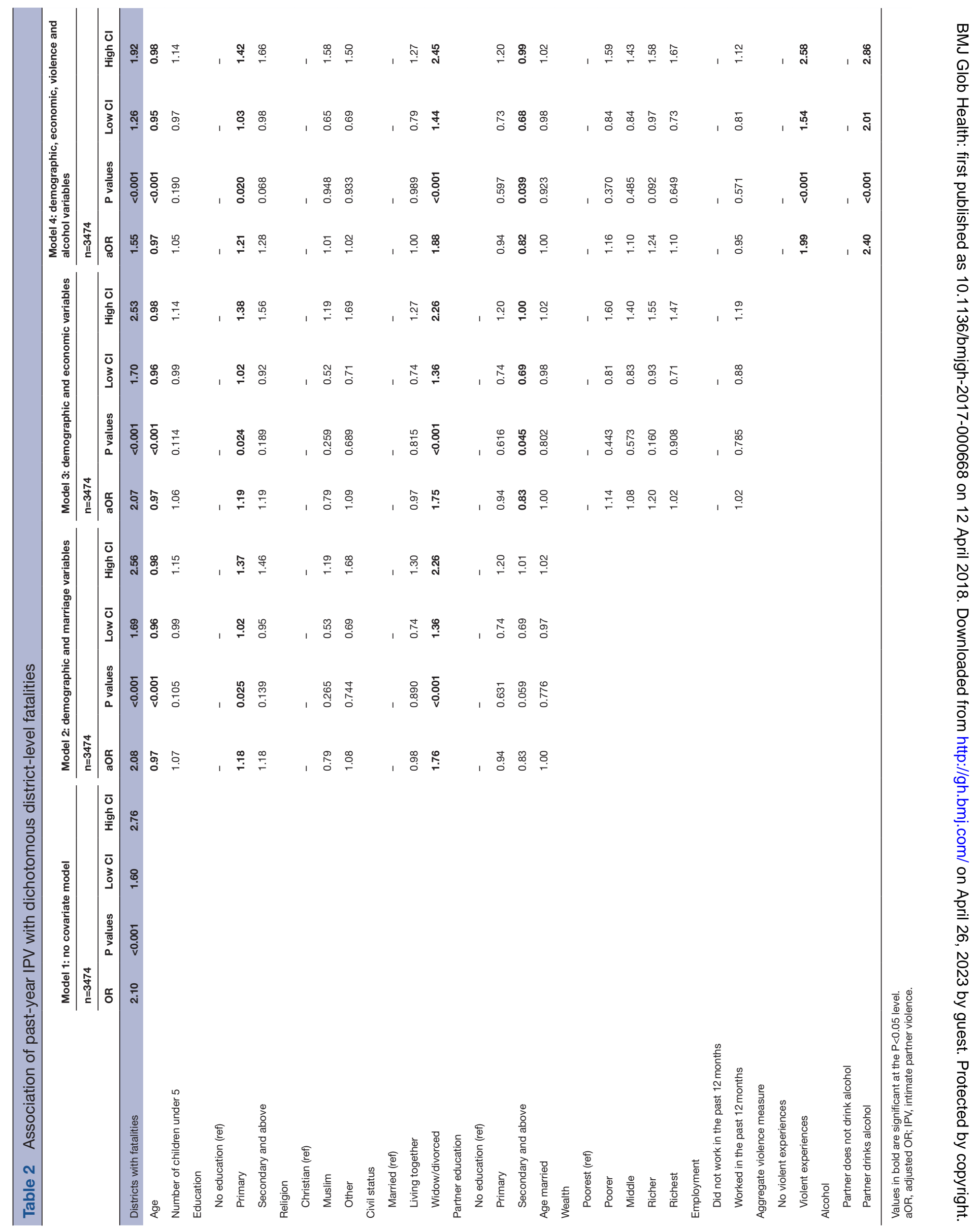




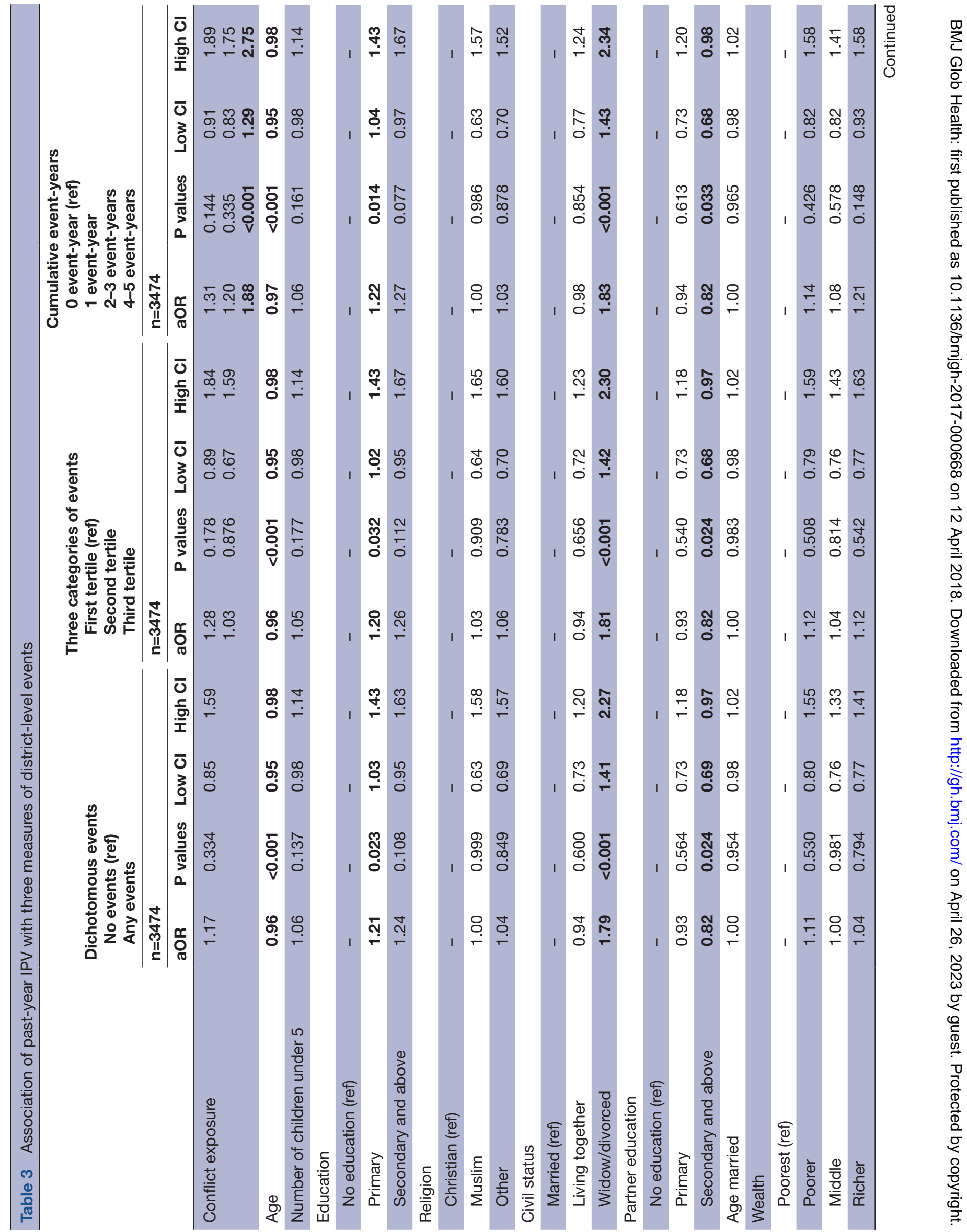




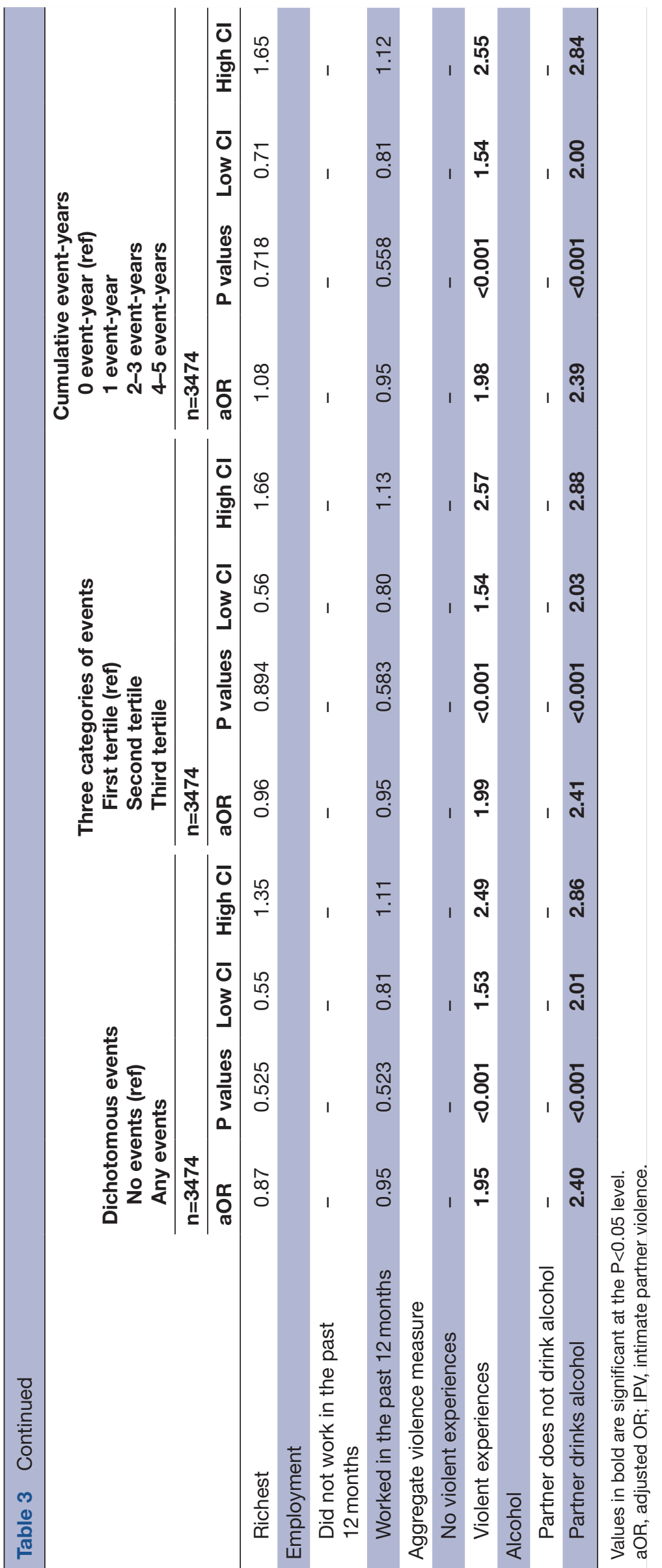

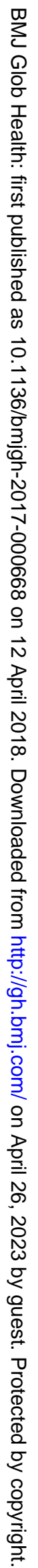


both men and women. ${ }^{5}$ Women were more likely to be victims of IPV, and men were more likely to perpetrate IPV for every war-related trauma they experienced. These strong associations almost a decade after the war speak to the lasting impact of collective trauma on violence in the home. While previous work focuses on drawing links between conflict and IPV at the individual level, this analysis demonstrates that conflict at the district level is also associated with higher rates of IPV. It is not clear whether this effect is driven by the fact that individuals in districts with conflict fatalities are directly affected by war and thus have the risk factors identified by Vinck and Pham, or whether there are more subtle and indirect processes at play.

Beyond the Liberian context, several surveys have documented the link between political violence and IPV in places as diverse as Côte d'Ivoire, Myanmar, Uganda and Afghanistan. ${ }^{2-4} 1725-27$ These studies point to a persistent link between war and IPV, but also highlight important nuances as well. Refugee women on the Thailand-Myanmar border reported rates of IPV six times higher than non-refugee women. ${ }^{3}$ This work suggests that perhaps layered vulnerabilities, such as displacement and experiencing personal war trauma, may have an interaction effect leading to far higher rates of IPV than experiencing only one event alone. This study is not able to look at the difference in experiences of women directly affected by war versus those not directly affected. However, this would be a valuable area for future inquiry. Future DHS surveys in postconflict contexts might consider adding a small module on war experiences and displacement to add to this literature. In addition, IPV is one of many forms of interpersonal violence-which also encompasses non-partner sexual violence, school violence and child abuse to name only a few. Research could be done to explore whether political violence affects other forms of aggression as well.

Further work is needed to explore the pathways through which political violence may influence IPV in the postconflict phase. The authors hypothesise that there are both 'direct' pathways for the spread of violence; for instance, conflict-affected districts may have greater numbers of former combatants who may normalise violent behaviour and may have high rates of drug and alcohol abuse. It is also posited, however, that there are also myriad 'indirect' pathways for conflict to influence IPV. These pathways may include societal habituation to conflict, a weakening of social support structures, crises in family roles and responsibilities, and disruption of peaceful conflict resolution practices. Some variables, for instance, alcohol use, are well-documented contributors to IPV independent of conflict. ${ }^{28}$ However, the trauma of experiencing conflict may also lead to behaviours that are associated with higher levels of IPV—-such as higher rates of alcohol abuse. Thus, alcohol may mediate, but not fully account for, the relationship between conflict and IPV—as suggested by the model results. The current work does not examine which pathways may be most important in this association. However, this is a promising avenue for future investigation.

Previous literature has also explored the role of violent masculinities before, during ${ }^{29}{ }^{30}$ and after war. ${ }^{31}{ }^{32}$ It is possible that those districts experiencing higher rates of conflict-related fatalities may also have had hypermasculinised or violence-permissive cultures before hostilities that also predisposed them to higher rates of IPV after war. While the models presented do adjust for childhood levels of violence, this may not fully capture the extent to which a district may have a culture permissive towards violence. There is some literature to suggest that societal-level norms about violence may influence violence experienced by individuals living in those areas. A population-based study from Nigeria found that administrative areas with social norms that were more permissive towards spousal violence were correlated with higher reported rates of women's reports of physical and sexual IPV ${ }^{33}$ Social attitudes towards the acceptability of violence may indeed underlie the propensity for violence at the outbreak of war and higher rates of violence against women after war. A dearth of longitudinal data tracking attitudes towards, and experiences of, violence before, during and after conflict make it difficult to test the extent to which permissive attitudes towards violence may also underlie the outbreak of political conflict. However, this question opens intriguing avenues for future work.

\section{Limitations}

IPV, fundamentally, is about interactions between dyads of people. Because no data were collected on men's experiences, this work was only able to examine the experiences of women. A further limitation of this work is that it is unable to explore the pathways through which spread of violent behaviour occurs. Qualitative work with perpetrators, victims and service providers could help illuminate this link. Additionally, we lacked important information on other features of districts including availability of health clinics, levels of unemployment and the condition of infrastructure, which could also be informative. While this study makes use of available data by relying on outcome data collected following exposure data, several limitations persist in understanding causal pathways. Longitudinal data would be optimal though challenging in a conflict setting. Additionally, the limited number of districts with conflict fatalities made it difficult to examine a dose-response relationship. Our analysis of district-level conflict masks important heterogeneity within district. Districts were chosen as significant units because they represented heterogeneous units that still had adequate sample size. It would also be helpful to understand subdistrict effects or at whether individuals within a certain radius of conflict are more at risk for future violence. Finally, the 5-year time lag between the civil war and the DHS may have attenuated the association between IPV and the conflict measures. 


\section{Public health implications}

Our study fills an important void in the literature by showing that district-level political violence is associated with increased risk of IPV at the individual level, even years after the cessation of political conflict. We extend results from two previous unpublished studies from Rwanda and Peru, which found that individuals living in places affected by violent conflict were at higher risk for IPV. ${ }^{17} 18$ This paper also is the first to our knowledge clarify the types of conflict violence most detrimental for future outcomes. Efforts to quantify the severity of war have often relied purely on measures of mortality. ${ }^{34-36}$ This analysis hypothesised that conflict fatalities are the most definitive and violent measure of armed conflict, and therefore served as the primary predictor. However, three other measures of conflict-dichotomous events, three categories of events and cumulative conflict yearswere also assessed in follow-up sensitivity analyses. As predicted, districts with any versus no conflict fatalities had the strongest association with postconflict violence. However, districts with 4-5 cumulative years of violence also had higher rates of postconflict violence. Non-fatal events, whether parsed as a dichotomous or as a no, medium and high measure, were not significantly associated with IPV.

A troubling implication of the work on IPV and war is that conflict may create cohorts of individuals more likely to both perpetrate and experience violence. Previous work has clearly shown a link between children's exposure to IPV within the household and future perpetration and victimisation. ${ }^{37}$ This means that having a group of individuals exposed to mass violence may set off a ripple effect across generations.

Understanding the link between war and IPV will be critical for disrupting cycles of violence both immediately following and long after peace is declared. The evidence of a consistent link between IPV and political violence underscores the need for better response to this issue. This is particularly important because studies in both war-time and peace-time contexts have shown that exposure to violence, especially as an adolescent, can make one more likely to perpetrate or experience violence throughout the life-cycle and to pass on this legacy of behaviours to one's children. ${ }^{37}$

IPV represents a complex problem that requires intervention at multiple levels. Scholarship emphasises the need for interventions that focus on at-risk populations early in life, both for primary and secondary prevention programmes. Interventions that target mental health issues, substance abuse and aggressive behaviour in an integrated way may be most effective. ${ }^{38}{ }^{39}$ Addressing IPV after conflict requires even more specialised approaches. Postconflict peace-building and reconciliation efforts should find ways to explicitly incorporate IPV and domestic violence messaging. Trauma-healing programmes with former combatants and highly affected communities can target the problem of aggression within the home and should focus on conflict resolution, de-escalation and behaviour modification. Involving women in peace processes and reconstruction efforts can better ensure that issues important to women, for instance IPV, stay on the agenda and are addressed after conflict. Programming that promotes women's empowerment and raises awareness of IPV, and the services that are available to address this issue, could help mitigate possible increases in this form of violence during and after instability.

Combining multiple interventions to address the manifold impact of war is critical for achieving holistic and long-term change. Some of the most effective approaches described in the Democratic Republic of the Congo merge economic interventions, individual and family counselling, and community messaging. ${ }^{40} 41$

These results contribute to a growing literature that documents the link between political violence and IPV and are among the first efforts to use a multilevel modelling approach to explore this issue. Understanding the extent to which interpersonal violence may increase during and after conflict can help local health systems and civil society, including religious organisations and non-governmental organisations, anticipate increases in interpersonal violence. It is possible that higher rates of postconflict violence are an unrecognised problem that impedes recovery. By acknowledging and addressing these problems, communities can more effectively rebuild.

Contributors JTDK conducted the data analysis, interpreted the results and drafted the manuscript for this research. EC provided detailed guidance on the statistical methods, contributed to interpretation of results and write-up, and gave substantive feedback on the manuscript drafts. WCR provided expertise on conflict and postconflict research, contributed to interpretation of results and write-up, and gave substantive feedback on the manuscript drafts. MRD provided overall guidance and mentorship for this project, contributed to interpretation of results and write-up, and significantly contributed to the drafting and editing of the manuscript.

Funding This research received no specific grant from any funding agency in the public, commercial or not-for-profit sectors.

Competing interests None declared.

Patient consent Not required.

Ethics approval Johns Hopkins University exempted this study from full IRB review.

Provenance and peer review Not commissioned; externally peer reviewed.

Data sharing statement № additional data are available.

Open Access This is an Open Access article distributed in accordance with the Creative Commons Attribution Non Commercial (CC BY-NC 4.0) license, which permits others to distribute, remix, adapt, build upon this work non-commercially, and license their derivative works on different terms, provided the original work is properly cited and the use is non-commercial. See: http://creativecommons.org/ licenses/by-nc/4.0/

(c) Article author(s) (or their employer(s) unless otherwise stated in the text of the article) 2018. All rights reserved. No commercial use is permitted unless otherwise expressly granted.

\section{REFERENCES}

1. Pettersson T, Wallensteen P. Armed conflicts, 1946-2014. J Peace Res 2015;52:536-50.

2. Saile R, Neuner F, Ertl V, et al. Prevalence and predictors of partner violence against women in the aftermath of war: a survey among couples in northern Uganda. Soc Sci Med 2013;86:17-25. 
3. Falb KL, McCormick MC, Hemenway D, et al. Violence against refugee women along the Thai-Burma border. Int $J$ Gynaecol Obstet 2013;120:279-83.

4. Gupta J, Reed E, Kelly J, et al. Men's exposure to human rights violations and relations with perpetration of intimate partner violence in South Africa. J Epidemiol Community Health 2012;66:e2.

5. Vinck P, Pham PN. Association of exposure to intimate-partner physical violence and potentially traumatic war-related events with mental health in Liberia. Soc Sci Med 2013;77:41-9.

6. World Health Organization. Global and regional estimates of violence against women: prevalence and health effects of intimate partner violence and non-partner sexual violence. 2013. http://www.who. int/reproductivehealth/publications/violence/9789241564625/en/ (accessed 31 Oct 2017).

7. Devries KM, Mak JY, García-Moreno C, et al. Global health. The global prevalence of intimate partner violence against women. Science 2013;340:1527-8.

8. Campbell JC. Health consequences of intimate partner violence. Lancet 2002;359:1331-6.

9. Decker MR, Peitzmeier S, Olumide A, et al. Prevalence and health impact of intimate partner violence and non-partner sexual violence among female adolescents aged 15-19 years in vulnerable urban environments: a multi-country study. $J$ Adolesc Health 2014;55:S58-S67

10. Jewkes RK, Dunkle K, Nduna M, et al. Intimate partner violence, relationship power inequity, and incidence of HIV infection in young women in South Africa: a cohort study. Lancet 2010;376:41-8.

11. Miller E, Decker MR, McCauley HL, et al. Pregnancy coercion, intimate partner violence and unintended pregnancy. Contraception 2010;81:316-22.

12. Mitchell J, Wight $\mathrm{M}$, Van Heerden $\mathrm{A}$, et al. Intimate partner violence, HIV, and mental health: a triple epidemic of global proportions. Int Rev Psychiatry 2016;28:452-63.

13. Pallitto CC, García-Moreno C, Jansen HA, et al. Intimate partner violence, abortion, and unintended pregnancy: results from the WHO Multi-country Study on Women's Health and Domestic Violence. Int J Gynaecol Obstet 2013;120:3-9.

14. Stockman JK, Lucea MB, Campbell JC. Forced sexual initiation, sexual intimate partner violence and HIV risk in women: a global review of the literature. AIDS Behav 2013;17:832-47.

15. Clark CJ, Everson-Rose SA, Suglia SF, et al. Association between exposure to political violence and intimate-partner violence in the occupied Palestinian territory: a cross-sectional study. Lancet 2010;375:310-6.

16. Gupta J, Acevedo-Garcia D, Hemenway D, et al. Premigration exposure to political violence and perpetration of intimate partner violence among immigrant men in Boston. Am J Public Health 2009;99:462-9.

17. Gallegos JV, Gutierrez IA. The effect of civil conflict on domestic violence: the case of Peru. SSRN Electronic Journal 2011.

18. Janko M, Bloom S, Spencer J. Community exposure to violent conflict increases the risk of intimate partner violence in Rwanda. Boston, MA: Population Association of America, 2014.

19. British Broadcasting Corporation website. Liberia country profile Overview. 2017. http://www.bbc.com/news/world-africa-13729504 (accessed 31 Oct 2017).
20. Central Intelligence Agency website. World factbook Liberia country profile. 2017. https://www.cia.gov/library/publications/the-worldfactbook/geos/print_li.html (accessed 31 Oct 2017).

21. United Nations. Security Council extends UN peace mission in Liberia for another year. 2017. http://www.un.org/apps/news/story. asp?NewsID=45888\#.V-0vgvkrLIU (accessed 31 Oct 2017).

22. Straus MA, Gelles RJ, Smith C. Physical violence in American families: Risk factors and adaptations to violence in 8,145 families. New Brunswick, NJ: Transaction Publishers, 1990.

23. StataCorp LP. Stata/SE 14.0 for Windows. College Station, TX: StataCorp LP, 2011.

24. Rabe-Hesketh S, Skrondal A, Pickles A, et al. Berkeley division of biostatistics working paper series. U.C. Berkeley: Division of Biostatistics Working Paper Series, 2014. Working Paper 160.

25. Annan J, Brier M. The risk of return: intimate partner violence in northern Uganda's armed conflict. Soc Sci Med 2010;70:152-9.

26. Catani C, Jacob N, Schauer E, et al. Family violence, war, and natural disasters: a study of the effect of extreme stress on children's mental health in Sri Lanka. BMC Psychiatry 2008;8:33.

27. Hossain M, Zimmerman C, Kiss L, et al. Men's and women's experiences of violence and traumatic events in rural Cote d'Ivoire before, during and after a period of armed conflict. British Medical Journal 2014:4:e003644.

28. Greene MC, Kane JC, Tol WA. Alcohol use and intimate partner violence among women and their partners in sub-Saharan Africa. Glob Ment Health 2017;4:e13.

29. Goldstein JS. War and gender. In: Ember CR, Ember M, eds. Encyclopedia of sex and gender. USA: Springer, 2003:107-16.

30. Yuval-Davis N. Gender and Nation. USA: SAGE Publications, 1997.

31. Aoláin FN, Cahn N, Haynes D. Masculinities and child soldiers in post-conflict societies. Masculinities and the Law: A Multidimensional Approach 2012;27:231.

32. Theidon K. Reconstructing masculinities: the disarmament, demobilization, and reintegration of former combatants in Colombia. Hum Rights Q 2009;31:1-34.

33. Linos N, Slopen N, Subramanian SV, et al. Influence of community social norms on spousal violence: a population-based multilevel study of Nigerian women. Am J Public Health 2013;103:148-55

34. Themnér L, Wallensteen P. Armed conflicts, 1946-2013. Journal of Peace Research 2014;51:541-54.

35. Global Burden of Armed Violence. Geneva declaration. Geneva, Switzerland: Global Burden of Armed Violence, 2011. (accessed 31 Oct 2017).

36. Gleditsch KS, Metternich NW, Ruggeri A. Data and progress in peace and conflict research. J Peace Res 2014;51:301-14.

37. Roberts AL, Gilman SE, Fitzmaurice G, et al. Witness of intimate partner violence in childhood and perpetration of intimate partner violence in adulthood. Epidemiology 2010;21:809-18.

38. Jewkes R. Intimate partner violence: causes and prevention. The Lancet 2002:359:1423-9.

39. Porter A. 'What is Constructed can be transformed': masculinities in post-conflict societies in Africa. Int Peacekeep 2013;20:486-506.

40. Kelly JT, Betancourt TS, Mukwege D, et al. Experiences of female survivors of sexual violence in eastern Democratic Republic of the Congo: a mixed-methods study. Confl Health 2011;5:25.

41. Kohli A, Makambo MT, Ramazani P, et al. A Congolese communitybased health program for survivors of sexual violence. Confl Health 2012;6:6. 